\title{
Cumulative glycaemia as measured by lens fluorometry: association with retinopathy in type 2 diabetes
}

\author{
I. C. Munch • M. Larsen • K. Borch-Johnsen • \\ C. Glümer • H. Lund-Andersen • L. Kessel
}

Received: 18 September 2010 / Accepted: 29 November 2010/Published online: 29 December 2010

(C) Springer-Verlag 2010

\begin{abstract}
Aims/hypothesis The aim of this study was to assess the association between lifelong cumulative glycaemia estimated by lens fluorometry and the presence of retinopathy in individuals with type 2 diabetes.

Methods We carried out a cross-sectional population-based study of 970 participants aged between 30 and 60 years, of which 170 were diagnosed with diabetes on screening (WHO 1999 criteria) and 35 had known type 2 diabetes. Procedures included clinical and laboratory examinations, non-invasive assessment of the intrinsic fluorescence of the lens of the eye, and seven-field fundus photography.

Results Retinopathy was found in 46 (22\%) of 205 participants with type 2 diabetes. In a logistic regression analysis controlling for age, sex and diabetes status (screen-
\end{abstract}

I. C. Munch $(\bowtie) \cdot$ M. Larsen · H. Lund-Andersen $\cdot$ L. Kessel

Department of Ophthalmology, Glostrup Hospital,

University of Copenhagen,

Nordre Ringvej 59,

2600 Glostrup, Denmark

e-mail: icm@dadlnet.dk

I. C. Munch • M. Larsen · H. Lund-Andersen

Faculty of Health Sciences, University of Copenhagen,

Copenhagen, Denmark

K. Borch-Johnsen $\cdot$ H. Lund-Andersen

Steno Diabetes Center,

Gentofte, Denmark

K. Borch-Johnsen

Faculty of Health Sciences, University of Aarhus,

Aarhus, Denmark

C. Glümer

Research Centre for Prevention and Health, Glostrup Hospital,

Glostrup, Denmark detected or known), a two-fold increase in lens fluorescence increased the odds for retinopathy by 3.46 (95\% CI $1.25-$ $9.55, p=0.017)$. The association was marginally significant (OR 3.00 [95\% CI 1.00-9.01], $p=0.050$ ) when also adjusted for smoking, systolic blood pressure, body mass index and $\mathrm{HbA}_{1 \mathrm{c}}$.

Conclusions/interpretation Diabetic retinopathy was related to cumulative lifelong glycaemia as estimated by lens fluorometry in participants with type 2 diabetes. This supports the hypothesis that retinopathy is a marker of lifelong elevated glycaemia as well as of the unknown, prediagnostic duration of type 2 diabetes. The powerful association between lens fluorescence and retinopathy underscores the importance of strict long-term glycaemic control in the prevention of retinopathy in people with diabetes.

Keywords Cumulative glycaemia - Lens fluorescence · Retinopathy Type 2 diabetes

\author{
Abbreviations \\ ETDRS Early Treatment Diabetic Retinopathy Study \\ FPG Fasting plasma glucose
}

\section{Introduction}

A major challenge in the management of patients with type 2 diabetes is that the disease can be asymptomatic for many years until it is diagnosed following the appearance of long-term complications. Risk factor analysis for complications of type 2 diabetes suffers from an inherent inability to account for the duration of diabetes, which cannot be assessed retrospectively. An index of lifelong cumulative 
glycaemia can be obtained by measuring fluorescence in the lens of the eye. The bulk of the lens proteins are never lost or replaced [1] and therefore accumulate fluorescent adducts in proportion to age and glycaemia after glucose has reacted with the lens protein amino residuals [2]. The same spontaneous reaction leads to the formation of $\mathrm{HbA}_{1 \mathrm{c}}$ [2].

Lens fluorescence has been shown to increase with age [3], duration of diabetes [3, 4], glycaemia [5] and smoking [6]. The presence of retinopathy in a clinic-based population of patients with variable diabetes duration and type has been found to be associated with higher levels of fluorescence measured by a method not correcting for intrinsic light absorption [4]. The aim of the present study was to investigate if retinopathy is associated with lens fluorescence in a population-based study of primarily newly diagnosed individuals with type 2 diabetes.

\section{Methods}

Study participants were recruited from the baseline visit of the Inter99 study, a randomised non-pharmacological intervention study of ischaemic heart disease prevention [7]. An age- and sex-stratified random sample of 12,934 eligible individuals aged 30-60 years was invited to participate, of whom 6,906 (53.4\%) volunteered. In brief, the Inter99 Eye study examined 970 participants from the Inter99 study between 1999 and 2001, including a random sample of 563 participants and a skewed sample of 407 participants with a high-risk profile defined by a compound cardiovascular risk score [8]. Diabetes-related data were available for 937 participants. The present analysis excluded six participants, three with type 1 diabetes (serum C-peptide $<100 \mathrm{pmol} / \mathrm{l}$ ) and three with ungradeable or missing fundus photographs, thus leaving 205 participants with type 2 diabetes for analysis. In 17 participants lens fluorometry was unavailable (lens opacities in three, instrument failure in 14).

All participants gave their written informed consent. The protocols were in accordance with the Helsinki Declaration and approved by the local medical ethics committees. The Inter99 study was registered in 2005 at ClinicalTrials.gov (registration no. NCT00289237).

After pupil dilation, participants underwent an ophthalmic examination including cataract assessment and 7-field non-stereoscopic 60-degree digital fundus photography (TRC-50X camera; Topcon, Tokyo, Japan) with a 1,024× 1,024 pixel CV-1000 back-piece (AngioVision 1000, MediVision, Tel Aviv, Israel) in red-free illumination (Wratten 54 filter; Eastman Kodak, Rochester, NY, USA) and fovea-centred stereoscopic colour diapositive photography (Ektachrome Elite 100; Eastman Kodak). Fundus characteristics were assessed by an ophthalmologist (I. C. Munch) masked to all systemic variables. The photographs were graded for retinal lesions and classified according to the Early Treatment Diabetic Retinopathy Study (ETDRS) retinopathy severity scale. The maximum grade in any of the seven standard fields in either of a participant's eyes defined the participant's retinopathy level.

Lens fluorescence was measured using a non-invasive ocular fluorometer (Fluorotron, OcuMetrics, Mountain View, CA, USA). Excitation was from 430 to $490 \mathrm{~nm}$ and detection 530-630 nm. Lens fluorescence was measured along the optical axis of the eye and measurements were corrected for loss of light by absorption in the lens. The mean of six scans, three per eye, was used in analysis. All fluorescence values were calibrated to an external solution of fluorescein and expressed as equivalent concentration of fluorescein in $\mathrm{ng} / \mathrm{ml}$ ( $\mathrm{ng} \mathrm{f}-\mathrm{eq} / \mathrm{ml}$ ). The coefficient of variation for repeated measurements of lens fluorescence is below $3 \%$ [3].

Participants were defined as having diabetes mellitus when fasting plasma glucose (FPG) was $7.0 \mathrm{mmol} / \mathrm{l}$ or higher or when $2 \mathrm{~h}$ plasma glucose during the oral glucose tolerance test was $11.1 \mathrm{mmol} / 1$ or higher or if participants responded to the study questionnaire that they had been diagnosed with diabetes by a physician.

The lower of two blood pressure recordings made after at least $5 \mathrm{~min}$ of rest was used for data analysis. Smoking was assessed by questionnaire, daily smoking being defined as an average consumption of $\geq 7 \mathrm{~g}$ of tobacco per day for more than 1 year.

Multiple logistic regression and general linear modelling (proc LOGISTIC and GLM, SAS software package version 9.1, SAS institute, Cary, NC, USA) was used for data analysis. Two-tailed $p$ values and $95 \%$ CIs were calculated by the use of Wald's test. Trends were estimated as the slope when categorical variables of interest were treated as quantitative variables. Log-linear assumptions were tested by likelihood ratio tests. Tests for interaction were performed by adding the cross product to the model.

\section{Results}

The 970 participants in the Inter99 Eye Study included 205 individuals with type 2 diabetes who had complete retinopathy data. Of these, $170(83 \%)$ had screen-detected diabetes and $35(17 \%)$ had known type 2 diabetes (Table 1). Some retinopathy (ETDRS level $\geq 15$ ) was found in 46 of the 205 participants (22\%) and mild retinopathy or worse (ETDRS level $\geq 35)$ was found in 10 of these 46 participants $(4.9 \%$ of all 205 participants with type 2 diabetes; Table 1).

Lens fluorescence was significantly associated with retinopathy. In a logistic regression model adjusting for age, sex and diabetes status (screen-detected or known), the OR for the presence of retinopathy was 4.82 (95\% CI 1.19- 
Table 1 Characteristics of included participants

\begin{tabular}{ll}
\hline Variable & $\begin{array}{l}\text { Inter99Eye Study participants } \\
\text { with type } 2 \text { diabetes }(n=205)\end{array}$ \\
\hline Age (years) & $51(7.1)$ \\
Male/female (\%) & $60 / 40$ \\
Danish nationality (\%) & 97 \\
BMI $\left(\mathrm{kg} / \mathrm{m}^{2}\right)$ & $30.8(6.0)$ \\
Systolic blood pressure (mmHg) & $142(18.1)$ \\
Diastolic blood pressure (mmHg) & $88(11.0)$ \\
Hypertension $(\%)^{\mathrm{a}}$ & 92 \\
Smoking daily $(\%)$ & 38 \\
Total cholesterol (mmol/l) & $5.9(1.1)$ \\
LDL-cholesterol (mmol/l) & $3.8(1.0)$ \\
Triacylglycerol (mmol/1) & $1.8(1.3)$ \\
Low HDL-cholesterol ${ }^{\mathrm{b}}(\%)$ & 41 \\
HbA ${ }_{1 \mathrm{c}}(\%)$ & $6.9(1.6)$ \\
FPG (mmol/l) & $7.1(2.1)$ \\
Known diabetes $(\%)$ & 17 \\
Duration of known diabetes (years) & $4.0(5.0)$ \\
Retinopathy $[n(\%)]:$ & \\
ETDRS $\geq 15$ & $46(22)$ \\
ETDRS $\geq 35$ & $10(4.9)$ \\
Lens fluorescence (mg f-eq/ml) & $673(310)$ \\
\hline
\end{tabular}

Data are presented as mean (standard deviation) or proportions except for triacylglycerol, FPG, duration of diabetes and lens fluorescence, which are presented with median and interquartile range because of a skewed distribution

${ }^{a}$ Hypertension is defined as systolic blood pressure $\geq 130 \mathrm{mmHg}$ or diastolic blood pressure $\geq 80 \mathrm{mmHg}$

${ }^{\mathrm{b}}<1.0 \mathrm{mmol} / \mathrm{l}(40 \mathrm{mg} / \mathrm{dl})$ in men and $<1.3 \mathrm{mmol} / 1(50 \mathrm{mg} / \mathrm{dl})$ in women

${ }^{\mathrm{c}}$ Data from 33 participants with known type 2 diabetes and information on the duration of diabetes

19.47) in participants in the top quartile of the lens fluorescence distribution compared with participants in the lowest quartile of the lens fluorescence distribution (Table 2). Including the logarithm of lens fluorescence as a quantitative trait in the model resulted in a better fit. A twofold increase in lens fluorescence raised the odds of having retinopathy by $3.46(95 \%$ CI $1.25-9.55, p=0.017)$ (Table 2). The effect of lens fluorescence on retinopathy persisted in a multivariate analysis adjusting also for smoking, systolic blood pressure, BMI and $\mathrm{HbA}_{1 \mathrm{c}}$, (OR 3.00 [95\% CI 1.00-9.01, $p=0.050]$ per twofold increase in the level of lens fluorescence) (Table 2). The logarithm of lens fluorescence increased with increasing $\mathrm{HbA}_{1 \mathrm{c}}(p=$ 0.0055 ) when controlling for age, sex, diabetes status and smoking (data not tabulated). No significant interaction between lens fluorescence and other variables was observed in any of the analyses.
The prevalence of retinopathy was higher in participants with known diabetes $(46 \%)$ than in participants with screen-detected diabetes (18\%) (Table 2). No significant interaction was observed between diabetes status (known vs screen-detected) and other tested variables. Female sex was protective of retinopathy, the effect being largely unaltered in the multivariate analysis (Table 2). Systolic blood pressure was associated with the presence of retinopathy and the association remained significant in a multivariate analysis (Table 2).

\section{Discussion}

In the present study, lens fluorescence, a biomarker of lifelong cumulative glycaemia, was independently associated with diabetic retinopathy in participants with type 2 diabetes.

The strengths of the study include the use of grey-scale fundus photography and lens fluorometry based on a scanning technique. A limitation was that ascertainment of participants included a planned over-representation of participants with screen-detected diabetes; however, the effects of lens fluorescence and other risk factors on retinopathy were found not to depend on diabetes status. The power to exclude associations was limited by the total of 46 participants with retinopathy.

A previous study also found associations between retinopathy and lens fluorescence in eyes with clear lenses in both early- and late-onset diabetes [4]. However, it was not population-based and did not include information about metabolic control or use a referenced method of retinopathy grading.

There is well-established evidence that the presence of retinopathy is an independent risk factor for systemic vascular complications [9] in type 2 diabetes. It is of interest for the understanding of diabetes to determine whether this association is based on retinopathy being a marker of the unknown pre-diagnostic duration and severity of type 2 diabetes or on retinopathy being an independent marker of vascular frailty. We found a strong association between retinopathy and the level of lens fluorescence, indicating that true duration of diabetes and hyperglycaemia severity are important factors in the development of retinopathy. Lens fluorescence may potentially be used in future studies to account for the unknown pre-diagnostic duration of type 2 diabetes.

Increased systolic blood pressure and high levels of $\mathrm{HbA}_{1 \mathrm{c}}$ are known risk factors for diabetic retinopathy [10] but in this study only increased systolic blood pressure was associated with retinopathy, either because study power was limited or because lens fluorescence was a better glycaemia biomarker. The prevalence of retinopathy was lower in 
Table 2 Lens fluorescence and other risk factors for retinopathy in participants with type 2 diabetes

\begin{tabular}{|c|c|c|c|}
\hline Variable & $\begin{array}{l}\text { Retinopathy }{ }^{\mathrm{a}} \\
\text { cases/number of participants (\%) }\end{array}$ & $\mathrm{OR}^{\mathrm{b}}(95 \% \mathrm{CI})$ & $\mathrm{OR}^{\mathrm{c}}(95 \% \mathrm{CI})$ \\
\hline \multicolumn{4}{|l|}{ Lens fluorescence } \\
\hline Per twofold increase & & $3.46(1.25-9.55)$ & $3.00(1.00-9.01)$ \\
\hline$p$ value & & 0.017 & 0.050 \\
\hline 1st quartile & $5 / 47(11)$ & 1 & 1 \\
\hline 2nd quartile & 9/47 (19) & $2.98(0.78-11.33)$ & $3.03(0.79-11.67)$ \\
\hline 3rd quartile & $10 / 47(21)$ & $3.21(0.78-13.11)$ & $2.88(0.69-12.02)$ \\
\hline 4th quartile & $16 / 47(34)$ & $4.82(1.19-19.47)$ & $3.69(0.86-15.74)$ \\
\hline$p$ value for trend & & 0.043 & 0.13 \\
\hline \multicolumn{4}{|l|}{ Age (years) } \\
\hline $30-39$ & $3 / 19(16)$ & 1 & 1 \\
\hline $40-49$ & $15 / 67(22)$ & $1.07(0.25-4.60)$ & $0.37(0.06-2.43)$ \\
\hline $50-60$ & 28/119 (24) & $1.19(0.29-4.86)$ & $0.29(0.038-2.21)$ \\
\hline$p$ value for trend & & 0.74 & 0.35 \\
\hline \multicolumn{4}{|l|}{ Sex } \\
\hline Male & $34 / 122(28)$ & 1 & 1 \\
\hline Female & $12 / 83(14)$ & $0.35(0.16-0.78)$ & $0.31(0.12-0.78)$ \\
\hline$p$ value & & 0.010 & 0.013 \\
\hline \multicolumn{4}{|l|}{ Status of diabetes } \\
\hline Screen-detected & $30 / 170(18)$ & 1 & 1 \\
\hline Known & $16 / 35(46)$ & $4.87(2.14-11.11)$ & $5.29(1.75-15.97)$ \\
\hline$p$ value & & 0.0002 & 0.0031 \\
\hline \multicolumn{4}{|l|}{ Daily smoking } \\
\hline No & $25 / 126(20)$ & 1 & 1 \\
\hline Yes & $21 / 78(27)$ & $1.16(0.56-2.41)$ & $1.26(0.53-2.98)$ \\
\hline$p$ value & & 0.68 & 0.61 \\
\hline \multicolumn{4}{|c|}{ Systolic blood pressure (mmHg) } \\
\hline$<130$ & $6 / 45(13)$ & 1 & 1 \\
\hline $130-139$ & $8 / 38(21)$ & $2.64(0.73-9.49)$ & $2.52(0.61-10.34)$ \\
\hline $140-159$ & $20 / 79(25)$ & $3.54(1.14-11.02)$ & $3.24(0.89-11.85)$ \\
\hline$\geq 160$ & $12 / 43(28)$ & $4.14(1.22-14.02)$ & $4.11(1.03-16.37)$ \\
\hline$p$ value for trend & & 0.020 & 0.046 \\
\hline \multicolumn{4}{|l|}{ BMI $\left(\mathrm{kg} / \mathrm{m}^{2}\right)$} \\
\hline$<25$ & $8 / 27(30)$ & 1 & 1 \\
\hline $25-29$ & $18 / 83(22)$ & $0.45(0.15-1.31)$ & $0.48(0.14-1.64)$ \\
\hline$\geq 30$ & 20/95 (21) & $0.49(0.17-1.37)$ & $0.59(0.16-2.11)$ \\
\hline$p$ value for trend & & 0.32 & 0.62 \\
\hline \multicolumn{4}{|l|}{$\mathrm{HbA}_{1 \mathrm{c}}(\%)$} \\
\hline$<6.0$ & 8/44 (18) & 1 & 1 \\
\hline $6.0-6.4$ & $9 / 52(17)$ & $0.63(0.21-1.92)$ & $0.84(0.26-2.69)$ \\
\hline $6.5-6.9$ & $8 / 44(18)$ & $0.63(0.20-2.00)$ & $0.55(0.14-2.12)$ \\
\hline$\geq 7.0$ & $21 / 65(32)$ & $1.08(0.38-3.01)$ & $0.75(0.23-2.51)$ \\
\hline$p$ value for trend & & 0.65 & 0.62 \\
\hline
\end{tabular}

${ }^{\text {a }}$ Retinopathy defined as ETDRS level $\geq 15$

${ }^{\mathrm{b}}$ Adjusted for age, sex and diabetes status (known/screen-detected)

${ }^{\mathrm{c}}$ Adjusted for all variables listed 
females compared with males. This may be a result of women diagnosed with screen-detected diabetes being healthier than their male counterparts, as the dose of glucose in the oral glucose tolerance test is unadjusted for differences in lean body weight.

In conclusion, this study provides evidence that diabetic retinopathy is related to cumulative lifelong glycaemia. It supports the hypothesis that retinopathy is a marker of elevated lifelong glycaemia and hence of the unknown, prediagnostic duration of type 2 diabetes. The powerful associations between lens fluorescence and retinopathy underscore the importance of strict glycaemic control in the prevention of diabetic retinopathy.

Duality of interest K. Borch-Johnson and C. Glümer hold shares in Novo Nordisk. The remaining authors have no duality of interest to declare.

\section{References}

1. Lynnerup N, Kjeldsen H, Heegaard S, Jacobsen C, Heinemeier J (2008) Radiocarbon dating of the human eye lens crystallines reveal proteins without carbon turnover throughout life. PLoS ONE 3:e1529
2. Monnier VM, Cerami A (1981) Nonenzymatic browning in vivo: possible process for aging of long-lived proteins. Science 211:491-493

3. Bleeker JC, van Best JA, Vrij L, van der Velde EA, Oosterhuis JA (1986) Autofluorescence of the lens in diabetic and healthy subjects by fluorophotometry. Invest Ophthalmol Vis Sci 27:791794

4. Sparrow JM, Bron AJ, Brown NA, Neil HA (1992) Autofluorescence of the crystalline lens in early and late onset diabetes. Br J Ophthalmol 76:25-31

5. Larsen M, Kjer B, Bendtson I, Dalgaard P, Lund-Andersen H (1989) Lens fluorescence in relation to metabolic control of insulin-dependent diabetes mellitus. Arch Ophthalmol 107:5962

6. Kessel L, Hougaard JL, Sander B, Kyvik KO, Sorensen TI, Larsen M (2002) Lens ageing as an indicator of tissue damage associated with smoking and non-enzymatic glycation - a twin study. Diabetologia 45:1457-1462

7. Jorgensen T, Borch-Johnsen K, Thomsen TF, Ibsen H, Glumer C, Pisinger C (2003) A randomized non-pharmacological intervention study for prevention of ischaemic heart disease: baseline results Inter99. Eur J Cardiovasc Prev Rehab 10:377-386

8. Kessel L, Hougaard JL, Mortensen C, Jorgensen T, LundAndersen H, Larsen M (2004) Visual acuity and refractive errors in a suburban Danish population: Inter99 Eye Study. Acta Ophthalmol Scand 82:19-24

9. Cheung N, Wong TY (2008) Diabetic retinopathy and systemic vascular complications. Prog Retin Eye Res 27:161-176

10. Klein R, Klein BE, Moss SE, Davis MD, DeMets DL (1984) The Wisconsin epidemiologic study of diabetic retinopathy. III. Prevalence and risk of diabetic retinopathy when age at diagnosis is 30 or more years. Arch Ophthalmol 102:527-532 